\title{
Why study multifractal spectra?
}

\author{
Peter Mörters \\ Department of Mathematical Sciences \\ University of Bath \\ Bath BA2 7AY, England
}

November 13, 2007

\begin{abstract}
We show by three simple examples how multifractal spectra can enrich our understanding of stochastic processes. The first example concerns the problem of describing the speed of fragmentation in a stick-breaking process, the second concerns the nature of a phase transition in a simple model of statistical mechanics, and the third example discusses the speed of emergence in Kingman's coalescent.
\end{abstract}

\section{Introduction}

I am often asked why I am interested in Hausdorff dimension. Are there any important problems that can be solved using Hausdorff dimension? Can Hausdorff dimension really add to our understanding of stochastic processes? I believe that the answer is yes to both questions, and in this article I attempt to give some evidence in the case of the second question, by means of three examples. I will focus on the notion of a multifractal spectrum or dimension spectrum, which in its broadest form refers to the Hausdorff dimension of a parametrised family of sets, seen as a function of the parameter.

The examples are chosen on the one hand for their relative simplicity, on the other hand to illustrate the diversity of shapes, which a multifractal spectrum can take. A common thread in all the examples is the notion of a tree, which either features prominently in the initial description or presents a very valuable reformulation of the model. Moreover, all our proofs rely, in some form or other, on one of the most beautiful and powerful ideas of probability theory, the concept of a martingale. Nevertheless, I will not give full details of any proofs in this review, but only sketch the basic ideas.

In the first example of this paper we look at the iterated breaking of a stick of unit length into a (random) finite number of parts. With every point on the stick we associate a fragmentation speed, measuring the rate at which the length of the part containing this point goes to zero. Attempting to plot the 
fragmentation speed as a function of the point position of the stick we are confronted with an extremely irregular (or fractal) function. A multifractal spectrum turns out to be exactly the right way to organise the information contained in this function in a comprehensible way.

In the second example we look at a simple model of a polymer in a random environment. We associate random weights to the vertices of a regular tree and model a polymer chain attracted by large weights and repelled by small weights by a path in the tree. More precisely, for fixed large $n$, we associate to each path $v$ from the root to the $n$th generation a probability proportional to $\exp \{\beta H(v)\}$ where $H(v)$ is the sum of the weights along $v$ and $\beta>0$ is an inverse temperature parameter. This model often has a phase transition, a sudden qualitative change as $\beta$ increases from zero to infinity, which is noticeable in the limiting behaviour of the normalisation factor, or partition function. In the absence of a spatial component in this model, the qualitative difference between the two phases is difficult to grasp. A multifractal spectrum helps attaching a physical meaning to this phase transition.

In the third example we look at a famous process arising in the context of mathematical biology. Kingman's coalescent describes the genealogy of a population in terms of a process with values in the set of partitions of $\mathbb{N}$ : Consider a population consisting at time one of infinitely many individuals, which are represented by the natural numbers. For any $s>0$ the individuals are then grouped into blocks $B \subset \mathbb{N}$ sharing the same ancestor at time $1-s$. This model has the interesting feature that at $s=0$ there is a transition from a partition consisting of infinitely many finite blocks to partitions consisting of finitely many infinite blocks. A multifractal spectrum allows us to better understand how this instant coalescence happens.

The first two examples represent the first steps in ongoing work with current $\mathrm{PhD}$ students of mine and I would like to thank them for permission to include this material here and for providing the illustrative pictures: The first example is drawn from joint work with Adam Kinnison, the second one from joint work with Marcel Ortgiese. Both will publish more substantial accounts of their work when the time is right, and I hope this article can serve as an advertisement for their work.

The third example was communicated to me by Julien Berestycki, and full details are yet to be written. It is an adaptation of results in Berestycki, Berestycki and Schweinsberg [2], which concerns the class of Beta-coalescents. The Kingman coalescent is a limiting case of the Beta-coalescents, which is different in some respects. Its advantage from our point of view is that its treatment can be based on more familiar concepts. Readers interested in the original result and a more sophisticated treatment of Beta-coalescents are recommended to consult [2], and to see also [5] and the contribution of Birkner and Blath in this volume for a survey of related results. 


\section{The speed of fragmentation in stick-breaking}

Suppose that $N$ is a nondegenerate random variable with values in the positive integers, and assume that

$$
\gamma(\beta):=\log \mathbf{E}\left[N^{1-\beta}\right]<\infty \quad \text { for every } \beta \in \mathbb{R} .
$$

We begin with a stick of length one, represented by the unit interval. At the first stage we sample $N$ and break this stick into $N$ sticks of length $1 / N$. At the $n$th stage we sample for every stick of the $(n-1)$ st stage an independent random variable $N$ and break this stick into $N$ further pieces of equal length. Hence, at any time $n$, we have a partition of the unit interval into a finite, random number of intervals (or sticks) of random length.

Having done this we can associate with every point $x \in[0,1]$ a decreasing sequence $\left(\ell_{1}, \ell_{2}, \ldots\right)$ where $\ell_{n}=\ell_{n}(x)$ is the length of the stick containing $x$ in the $n$th stage. The fragmentation speed at $x$ is

$$
f(x):=-\lim _{n \rightarrow \infty} \frac{1}{n} \log \ell_{n}(x),
$$

whenever this limit is defined. It is a natural problem to explore the nature of the random function $f$ for various distributions of $N$.

We first note that

$$
f(x)=\lim _{n \rightarrow \infty} \frac{1}{n} \sum_{j=1}^{n} \log N_{n}(x),
$$

where $N_{n}(x)$ is the number of pieces in which the stick containing $x$ is broken in the $n^{\text {th }}$ step. For any fixed $x$ the sequence $N_{1}(x), N_{2}(x), \ldots$ is i.i.d. and hence, by the law of large numbers, $f(x)=\mathbf{E}[\log N]$ almost surely. By Fubini's theorem we thus get

$$
0=\int_{0}^{1} \mathbf{E}|f(x)-\mathbf{E}[\log N]| d x=\mathbf{E} \int_{0}^{1}|f(x)-\mathbf{E}[\log N]| d x,
$$

and hence, almost surely,

$$
f(x)=\mathbf{E}[\log N] \quad \text { for Lebesgue-almost all } x .
$$

But the analysis does not end with the fact that $f$ is constant almost everywhere. The plot in Figure 1 reveals its fractal nature even in the case when $N$ is uniformly distributed. What one might guess from the picture is that $f$ is bounded from above and below, and that values above seem to be a lot more common than values below $\mathbf{E} \log N$. 


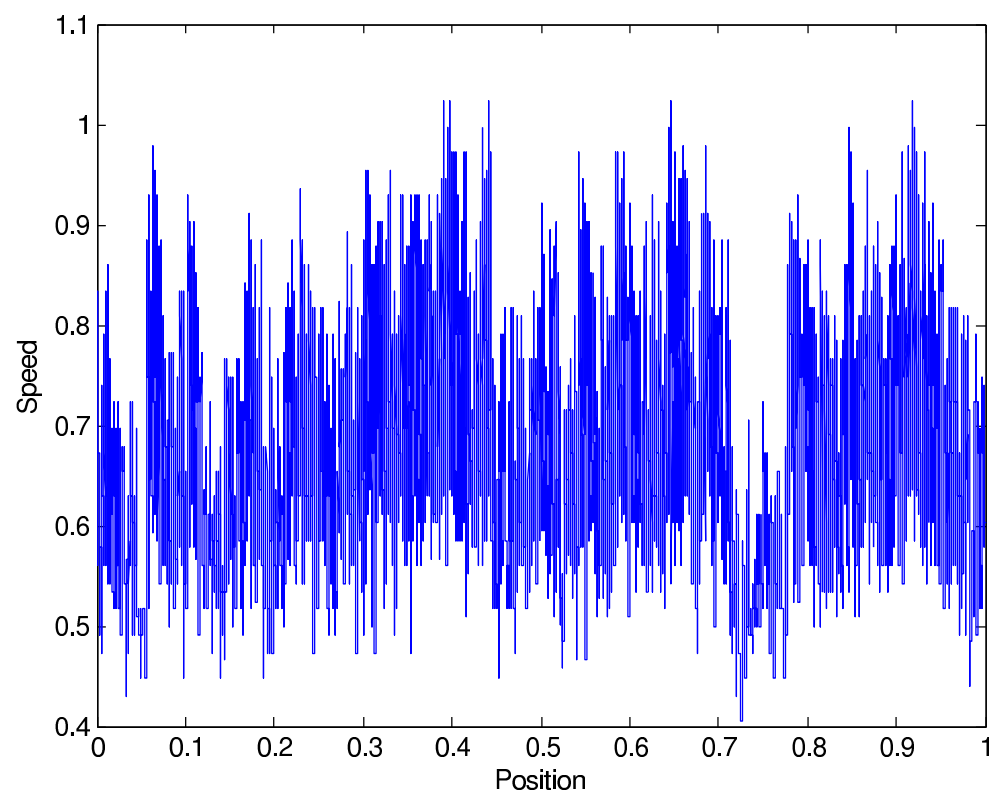

Figure 1: Plot of the speed in the case of the uniform distribution on $\{1,2,3\}$ using an approximation based on the fragmentation after 16 steps. The typical value of $f$ is here $\mathbf{E} \log N \approx 0.597$.

The interesting question is therefore: How frequent are the various values of $f$ ? Can we measure and compare the size of the sets

$$
\mathrm{S}(a):=\left\{x \in[0,1]:-\lim _{n \rightarrow \infty} \frac{1}{n} \log \ell_{n}(x)=a\right\}
$$

for all possible values of $a$ ? If this can be done, the nontrivial information contained in $f$ would take the form of a function mapping any possible value $a$ to the size of the set $\mathrm{S}(a)$.

The next theorem shows that this is indeed possible when Hausdorff dimension is used as the notion of size. The resulting (deterministic) function is a typical example of a multifractal spectrum, see Figure 2 for an example plot.

Theorem 1 (Kinnison). For every $a \geq 0$, almost surely

$$
\operatorname{dim} \mathrm{S}(a)=\frac{1}{a} \inf _{\beta}\{a \beta+\gamma(\beta)\}
$$

whenever the right hand side is nonnegative.

Remark: Our stick-breaking process is a discrete-time example of a random fragmentation process. A thorough multifractal analysis of continuous-time homogeneous fragmentation models has been performed by J. Berestycki [1]. Our result is not contained in his, and our proof uses a different setup, but there is still a great similarity of ideas. For further study in the mathematical theory of random fragmentation I recommend the recent book of Bertoin [3]. 


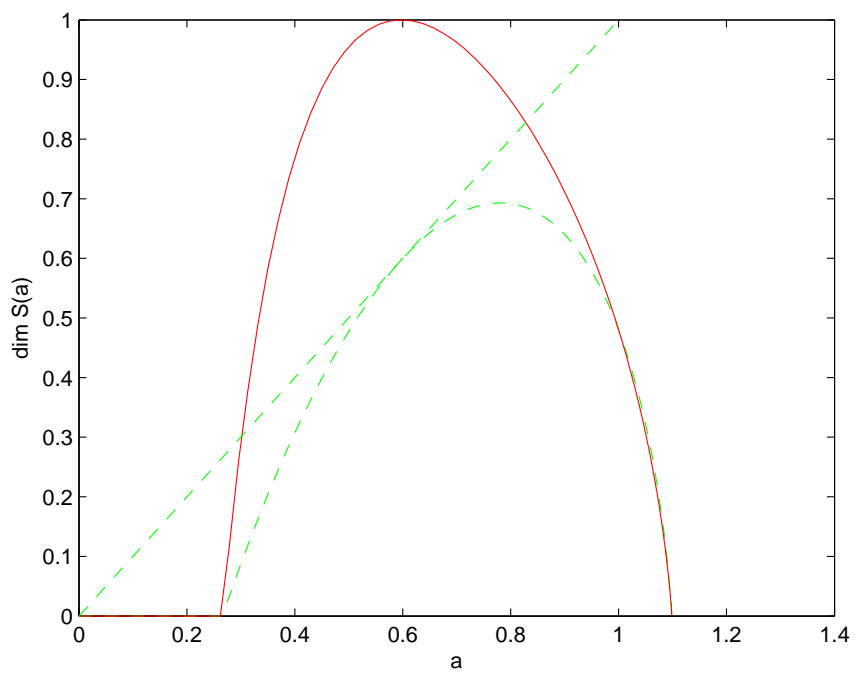

Figure 2: The multifractal spectrum for the stick-breaking process with $N$ uniformly distributed on $\{1,2,3\}$ is the bold curve, the dotted curves are the diagonal and the function $a \mapsto \inf _{\beta}\{a \beta+\gamma(\beta)\}$ included for comparison.

\section{The idea of the proof of Theorem 1}

We reinterpret the problem in terms of a tree, which will allow us to introduce the crucial objects in a natural manner. First, represent the closed unit interval $[0,1]$ as the root $\rho$ of the tree, then let $N(\rho)$ be the number of parts into which the unit interval is split, representing the new sticks as the children of the root. Continuing this process, any vertex $v$ in the $n$th generation of the tree represents a stick arising after $n$ breaking steps. Let $N(v)$ be the number of parts in which this stick is broken, represent the parts as the children of the vertex $v$, and continue ad infinitum.

Denote the resulting tree by $T$ and, for each vertex $v \in T$, denote by $|v|$ its generation and by $T(v)$ the tree of descendants of $v$, which is rooted in $v$. Obviously, $T$ is a Galton-Watson tree with offspring distribution given by the law of $N$. The rays in this tree are sequences of vertices $\left(v_{0}, v_{1}, v_{2}, \ldots\right)$ such that $v_{0}=\rho$ and $v_{i+1}$ is a child of $v_{i}$. The set of rays, called the boundary $\partial T$ of the tree, carries a metric structure given by the genealogical distance,

$$
d(u, v)=\exp \left\{-\min \left\{n \in \mathbb{N}: u_{n} \neq v_{n}\right\}\right\} \quad \text { for } u, v \in \partial T .
$$

There is a canonical mapping $\phi$ from the tree to the unit interval $[0,1]$ such that the sequence of nested closed subintervals represented by the vertices of a ray is mapped onto the unique point contained in every interval. Except on a countable set (the boundary points of the construction intervals) this mapping is invertible. Note however that the metric on the tree is not equivalent to the Euclidean metric on the interval. 
Now run a random walk on the tree $T$, starting at the root and moving at each step to each of the children of the current vertex with the same probability. The resulting random sequence $\left(X_{0}, X_{1}, \ldots\right)$ is a ray. Hence the distribution $\nu$ of this ray is a measure on $\partial T$ and it is easy to observe that it is mapped under $\phi$ onto the Lebesgue measure $\lambda$ on $[0,1]$. Therefore, $\phi$ is an isomorphism from the (random) measure space $(\partial T, \mathcal{B}$ orel,$\nu)$ to the (non-random) measure space $([0,1]$, Borel,$\lambda)$. Moreover, any random walk on $T$, which starts in the root and in each step moves from a vertex to one of its children, generates a measure on $\partial T$ and hence, via the mapping $\phi$, also on the interval $[0,1]$.

The key to the proof is the use of a family $\left\{M_{n}^{(\beta)}: n \in \mathbb{N}\right\}$ of nonnegative martingales, defined, for any $\beta \in \mathbb{R}$, by

$$
M_{n}^{(\beta)}=e^{-n \gamma(\beta)} \sum_{|v|=n} \prod_{j=0}^{n-1} N\left(v_{j}\right)^{-\beta} .
$$

Let $M^{(\beta)}(T):=\lim M_{n}^{(\beta)}$. Under our moment conditions this convergence holds in the $L^{1}$-sense and the limit is almost surely positive, see for example [17].

We now sketch the proof of the upper bound in Theorem 1. To describe an efficient covering of $\mathbf{S}(a)$, fix some large $m \in \mathbb{N}$ and interpret

$$
\mathcal{S}:=\left\{v \in T:|v|=n \geq m, \sum_{j=0}^{n-1} \log N\left(v_{j}\right) \approx a n\right\}
$$

as a collection of intervals. This collection covers $\mathrm{S}(a)$ and its $s$-value is

$$
\sum_{I \in \mathcal{S}}|I|^{s} \approx \sum_{n=m}^{\infty} \sum_{|v|=n} e^{-a n s} 1\left\{\sum_{j=0}^{n-1} \log N\left(v_{j}\right) \approx a n\right\} .
$$

Suppose for a moment that $a>\mathbf{E}[\log N]$. Let $\beta<0$ and, using Chebyshev's inequality, estimate the expected $s$-value of the covering from above by

$$
\sum_{n=m}^{\infty} e^{-n(a s-a \beta)} \mathbf{E} \sum_{|v|=n} \prod_{j=0}^{n-1} N\left(v_{j}\right)^{-\beta} .
$$

By the convergence results for $\left\{M_{n}^{(\beta)}: n \in \mathbb{N}\right\}$ the expectation is of order $e^{n \gamma(\beta)}$. Hence the expected $s$-value is finite if $s>\frac{1}{a}(\gamma(\beta)+a \beta)$. Optimising over $\beta<0$ gives the required upper bound. For the case $a<\mathbf{E} N$ the analogous argument can be performed choosing $\beta>0$.

For the sketch of the more delicate lower bound in Theorem 1 we introduce

$$
\text { SpinedTrees }=\{(T, v): v \in \partial T\},
$$

the space of trees endowed with a ray acting as a 'spine'. There is a canonical shift $\theta:$ SpinedTrees $\rightarrow$ SpinedTrees which maps $(T, v)$ to the tree $T\left(v_{1}\right)$ of 
descendants of the first vertex in the original spine, together with the trace $\left(v_{1}, v_{2}, \ldots\right)$ of the spine in this tree.

Denote by GW the distribution of our Galton-Watson tree. Given $T$, we select a spine $\left(X_{0}, X_{1}, \ldots\right)$ by following a random walk started at the root and, in each step, moving to each of the children $w$ of the current vertex with a probability proportional to $M^{(\beta)}(T(w))$. Let $\mu_{T}^{(\beta)}$ be the law of this spine in $\partial T$.

If $\beta \neq 0$, the measure $\mu_{T}^{(\beta)}$ makes a size-biased choice of the trees $T\left(v_{1}\right), T\left(v_{2}\right), \ldots$ along the spine, and hence the measure given by $\mu_{T}^{(\beta)}(d v) \mathrm{GW}(d T)$ is not shiftinvariant on SpinedTrees. However, it is not hard to show (see, e.g. [18] for similar arguments) that this size-bias can be compensated entirely by introducing the martingale limit $M^{(\beta)}(T)$ as a density for GW, i.e.

$$
\mu_{T}^{(\beta)}(d v) M^{(\beta)}(T) \mathrm{GW}(d T)
$$

is a shift-invariant and ergodic measure on SpinedTrees. The ergodic theorem now allows us to determine the speed of fragmentation as

$$
\begin{aligned}
f(\phi(v)) & =\lim _{n \rightarrow \infty} \frac{1}{n} \sum_{j=0}^{n-1} \log N\left(X_{j}\right)=\iint \log N(\rho) M^{(\beta)}(T) \mathrm{GW}(d T) \\
& =\frac{\mathbf{E}\left[N^{1-\beta} \log N\right]}{\mathbf{E}\left[N^{1-\beta}\right]} \quad \text { for } \mu_{T}^{(\beta)} \text {-almost every } v \text { and GW-almost every } T .
\end{aligned}
$$

Every subset of $[0,1]$ which has full measure for $\mu_{T}^{(\beta)} \circ \phi^{-1}$ has at least the Hausdorff dimension given by a lower bound on the local dimension in each point. The local dimension in $\phi(v)$ equals

$$
\operatorname{dim} \mu_{T}^{(\beta)} \circ \phi^{-1}(\phi(v))=\lim _{n \rightarrow \infty} \frac{\log \left(\prod_{j=1}^{n} \frac{M^{(\beta)}\left(T\left(v_{j}\right)\right)}{\sum^{(\beta)}(T(w))}\right)}{-\log \left(\prod_{j=0}^{n-1} N\left(v_{j}\right)\right)},
$$

where the sums in the denominators are over all siblings $w$ of the vertex in the argument of the numerator. Denoting $a(\beta)=\mathbf{E}\left[N^{1-\beta} \log N\right] / \mathbf{E}\left[N^{1-\beta}\right]$ and using the ergodic theorem and a small calculation, this limit equals

$$
\begin{aligned}
\frac{-1}{a(\beta)} & \iint \log \left(\frac{M^{(\beta)}\left(T\left(v_{1}\right)\right)}{\sum M^{(\beta)}(T(w))}\right) \mu_{T}^{(\beta)}(d v) M^{(\beta)}(T) \mathrm{GW}(d T) \\
& =\frac{1}{a(\beta)}\left(\log \mathbf{E}\left[N^{1-\beta}\right]+\beta a(\beta)\right),
\end{aligned}
$$

for $\mu_{T}^{(\beta)}$-almost every $v$ and GW-almost every $T$.

Hence we get a lower bound for the spectrum as

$$
\left.\operatorname{dim} S(a(\beta)) \geq \frac{1}{a(\beta)}(\gamma(\beta)+\beta a(\beta))\right) .
$$

As $a(\beta)=-\gamma^{\prime}(\beta)$, for any $a=a(\beta)$ the parameter $\beta$ is the minimiser in the variational problem characterising the spectrum. Hence this lower bound coincides with the upper bound, completing the sketch of the argument. 


\section{A polymer model in a random environment}

We look at a very crude model of a polymer in a disordered medium, which was introduced by Derrida and Spohn in [8]. The main interest here is to understand the effect of the disorder on the asymptotic behaviour of the polymer and the occurrence of a phase transition.

To describe the disorder we let $V$ be a random variable such that

$$
\phi(t):=\mathbf{E}\left[e^{t V}\right]<\infty \quad \text { for all } t>0
$$

Let $T$ be a binary tree with root $\rho$. We endow $T$ with a disordered medium $\mathcal{V}=(V(v): v \in T)$ by letting each random weight $V(v)$ be an independent copy of the random variable $V$. We identify vertices $v$ in the tree with the chain $\left(v_{0}, \ldots, v_{n}\right)$, starting from the root $v_{0}$ and ending at $v_{n}=v$, such that each $v_{i+1}$ is a child of $v_{i}$.

In the 'finite volume' setting, the polymers of length $n$ are given by the vertices $v \in T$ in the $n$th generation. For any inverse temperature $\beta>0$ the probability of a polymer $v$ of length $n$ is given by

$$
P^{(\beta)}(v)=\frac{1}{Z_{n}(\beta)} \exp \left\{\beta \sum_{j=1}^{n} V\left(v_{j}\right)\right\},
$$

with a normalisation factor

$$
Z_{n}(\beta)=\sum_{|v|=n} \exp \left\{\beta \sum_{j=1}^{n} V\left(v_{j}\right)\right\},
$$

which is called the partition function.

One expects in this and similar models that the behaviour of the polymer depends on the parameter $\beta$ in the following manner: If $\beta$ is small, we are in an entropy-dominated regime, where the fluctuations in $\mathcal{V}$ have no big influence and limiting features are largely the same as in the case of a uniformly distributed polymer. For large values of $\beta$ we may encounter an energy-dominated regime where, due to the disorder, the phase space breaks up into pieces separated by free energy barriers. Polymers then follow specific tracks with large probability, an effect often called localisation.

In such a simple model there are not too many features to distinguish the phases, and a crucial rôle is played by the free energy, defined as $\lim (\beta n)^{-1} \log Z_{n}(\beta)$. We further define, for any $\beta>0$,

$$
h(\beta)=\frac{1}{\beta} \log \mathbf{E} \exp \{\beta V+\log 2\}=\frac{1}{\beta} \log (2 \phi(\beta)) .
$$

As $\beta \mapsto \beta h(\beta)$ is strictly convex, one can see that $h^{\prime}$ has at most one positive root. If it exists, we define $\beta_{\mathrm{c}}>0$ to be this root, and we let $\beta_{\mathrm{c}}=\infty$ otherwise. 
Theorem 2 (Buffet, Patrick and Pulé [7]). Almost surely, the free energy is

$$
\lim _{n \rightarrow \infty} \frac{1}{\beta n} \log Z_{n}(\beta)= \begin{cases}h(\beta) & \text { if } \beta \leq \beta_{\mathrm{c}} \\ h\left(\beta_{\mathrm{c}}\right) & \text { if } \beta \geq \beta_{\mathrm{c}}\end{cases}
$$

Remark: At the critical temperature $1 / \beta_{\mathrm{c}}$ the model has a phase transition and, for low temperatures, it is in a frozen state. The two phases are often called the weak disorder phase $\left(\beta<\beta_{\mathrm{c}}\right)$, and the strong disorder phase $\left(\beta>\beta_{\mathrm{c}}\right)$.

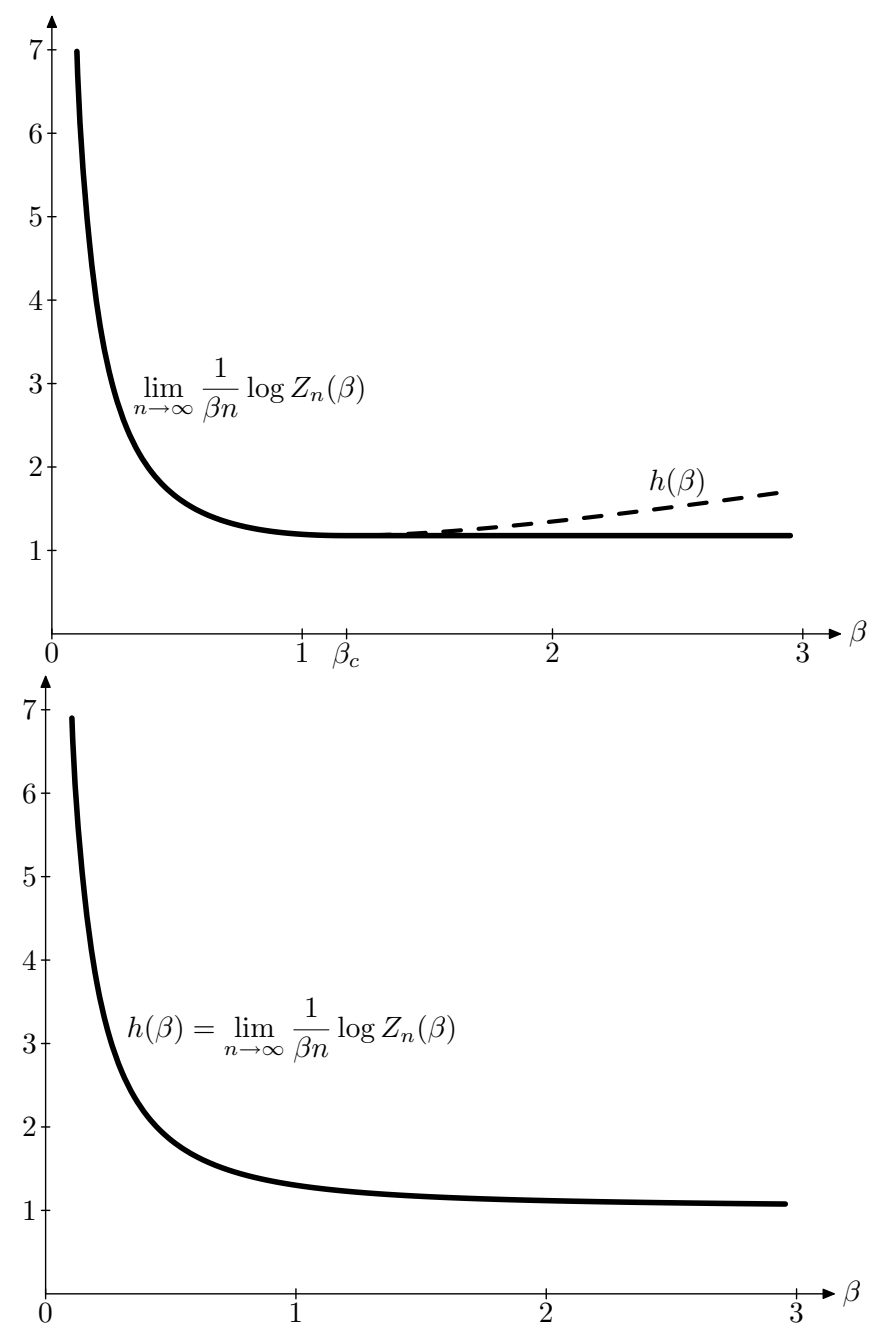

Figure 3: The free energy in the case of a standard normal distribution (top), where $\beta_{\mathrm{c}}<\infty$, and in the case of a binary distribution given by $\mathbb{P}\{V=1\}=$ $1-\mathbb{P}\{V=-1\}=p$ (bottom), where $\beta_{\mathrm{c}}=\infty$.

In the weak disorder phase we have

$$
\frac{1}{\beta n} \log Z_{n}(\beta) \sim \frac{1}{\beta n} \log \sum_{|v|=n} \mathbf{E} \exp \left\{\beta \sum_{j=1}^{n} V\left(v_{j}\right)\right\},
$$


and hence, at high temperatures, it may look like all polymers $v \in T$ with $|v|=n$ making the same contribution to $Z_{n}(\beta)$, namely the joint mean

$$
\mathbf{E} \exp \left\{\beta \sum_{j=1}^{n} V\left(v_{j}\right)\right\}=\phi(\beta)^{n} .
$$

In fact, this impression is wrong, and even at high temperatures only a vanishing proportion of the paths contribute to the free energy. The precise picture is conveyed by a multifractal spectrum.

To describe this spectrum we need a notion of a Hausdorff dimension of a tree. For the purpose of this article we use the growth rate

$$
\operatorname{dim}(\tilde{T}):=\lim _{n \rightarrow \infty} \frac{1}{n} \log \#\{v \in \tilde{T}:|v|=n\}
$$

as a notion of dimension and restrict the discussion to trees $\tilde{T}$ where this notion is well-defined. Let us emphasise that there is the more powerful concept of the branching number of a tree, introduced by Lyons, which is the appropriate way to measure the average number of children per vertex in an infinite tree. The logarithm of the branching number coincides with the Hausdorff dimension of the boundary of the tree, which carries a natural metric structure. For sufficiently regular trees this notion of dimension coincides with the growth rate, but in general the growth rate, if it exists, is the larger number. In this article we may restrict attention to the easier concept.

Theorem 3 (Ortgiese). Define $f:\left(0, \beta_{\mathrm{c}}\right) \rightarrow[0, \infty)$ by

$$
f(\beta)=\log 2+\log \phi(\beta)-\frac{\phi^{\prime}(\beta)}{\phi(\beta)} \beta .
$$

(a) For every $0<\beta<\beta_{\mathrm{c}}$, almost surely, there exists a tree $\tilde{T} \subset T$ with $\operatorname{dim}(\tilde{T})=f(\beta)$ such that

$$
\lim _{n \rightarrow \infty} \frac{1}{\beta n} \log \sum_{\substack{v \in \tilde{T} \\|v|=n}} \exp \left\{\beta \sum_{j=1}^{n} V\left(v_{j}\right)\right\}=h(\beta) .
$$

(b) Almost surely, for every $0<\beta<\beta_{\text {c }}$ and every tree $\tilde{T} \subset T$ such that $\operatorname{dim}(\tilde{T})<f(\beta)$, we have

$$
\lim _{n \rightarrow \infty} \frac{1}{\beta n} \log \sum_{\substack{v \in \tilde{T} \\|v|=n}} \exp \left\{\beta \sum_{j=1}^{n} V\left(v_{j}\right)\right\}<h(\beta) .
$$

Remark: If $0<\beta<\beta_{\mathrm{c}}$ the free energy is supported by a tree of dimension $f(\beta)$. The rays $v$ in this tree have the exceptional behaviour

$$
\lim _{n \rightarrow \infty} \frac{1}{n} \sum_{j=1}^{n} V\left(v_{j}\right)=a(\beta):=\frac{\phi^{\prime}(\beta)}{\phi(\beta)}>\mathbb{E} V .
$$




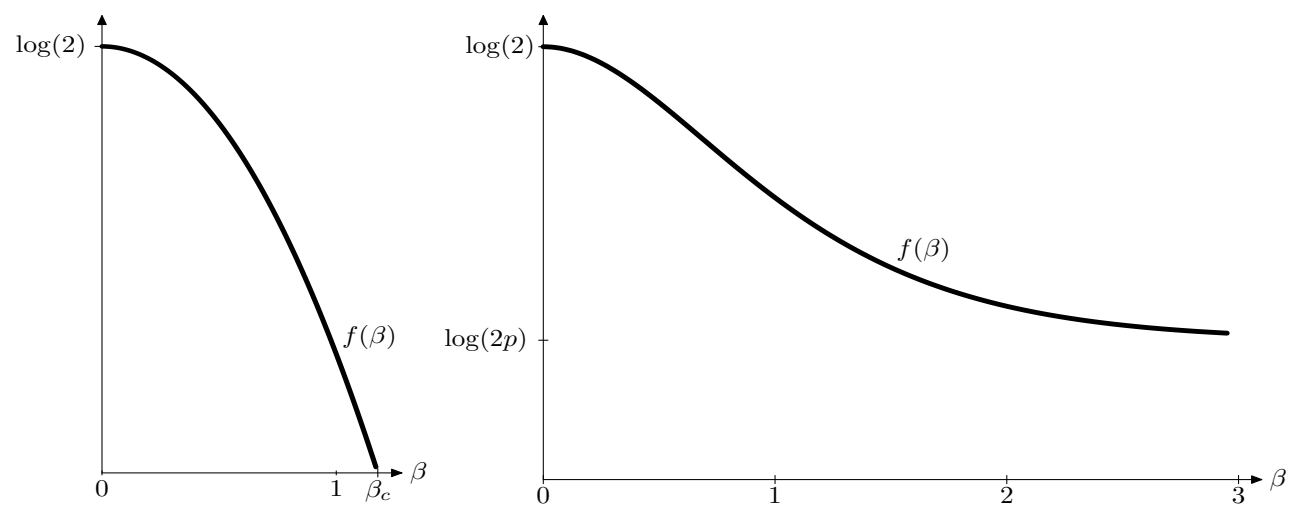

Figure 4: The dimension spectrum obtained by plotting the minimal dimension of a tree supporting the free energy at each $\beta<\beta_{\mathrm{c}}$ for a standard normal distribution (left) and at each $\beta<\infty$ for the binary distribution (right).

If $\beta \uparrow \beta_{\mathrm{c}}<\infty$ the dimension of this tree is going to zero and beyond the critical value there are no more rays with an average weight big enough to sustain a free energy of size $h(\beta)$. Instead, if $\beta>\beta_{\mathrm{c}}$ and $n$ large, a subexponential number of polymers of length $n$ with the maximal weight

$$
\frac{1}{n} \sum_{j=1}^{n} V\left(v_{j}\right) \approx a\left(\beta_{\mathrm{c}}\right)
$$

support $Z_{n}(\beta)$. We conjecture however, that these polymers are scattered all over the tree and therefore no subtree $\tilde{T} \subset T$ with $\operatorname{dim}(\tilde{T})<\log 2$ supports the free energy for all large $n$ in the low temperature regime.

\section{The idea of the proof of Theorem 3}

The key to the proof of Theorem 3 lies in the existence and fractal structure of the infinite volume Gibbs measure in the weak disorder regime. This object is of course also of independent interest.

To define the infinite volume Gibbs measure as a limit of the finite volume Gibbs measures we need to embed, for every $n \in \mathbb{N}$, the $n$th generation of the binary tree in its boundary $\partial T$. The easiest way to do this is by extending a vertex $w=\left(w_{0}, \ldots, w_{n}\right)$ uniquely to a ray $w^{+}=\left(w_{0}, w_{1}, \ldots\right)$ by letting $w_{i+1}$ be the left child of $w_{i}$ for any $i \geq n$. We can then define the finite volume Gibbs measures on the boundary $\partial T$ as

$$
\mu_{n}^{(\beta)}:=\frac{1}{Z_{n}(\beta)} \sum_{|w|=n} \exp \left\{\beta \sum_{j=1}^{n} V\left(w_{j}\right)\right\} \delta_{w^{+}},
$$

and, if possible, the infinite volume Gibbs measure as the almost-sure limit in the weak topology of measures

$$
\mu^{(\beta)}:=\lim _{n \rightarrow \infty} \mu_{n}^{(\beta)} .
$$


Martingales play the key role in the proof of existence of this measure in the weak disorder regime. Indeed, it is easy to verify that,

$$
M_{n}^{(\beta)}(v)=2^{-n} \phi(\beta)^{-n} \sum_{\substack{w \in T(v) \\|w|-|v|=n}} \exp \left\{\beta \sum_{j=|v|+1}^{|v|+n} V\left(w_{j}\right)\right\}
$$

defines a martingale $\left\{M_{n}^{(\beta)}(v): n \in \mathbb{N}\right\}$ for every $\beta>0$ and $v \in T$. Criteria for uniform integrability of these martingales can be found, for example, in [17] or [7]. They show that precisely if $\beta<\beta_{\text {c }}$ the martingales $\left\{M_{n}^{(\beta)}(v): n \in \mathbb{N}\right\}$ converge almost surely to a strictly positive limit, which we denote by $M^{(\beta)}(v)$.

Now focus on the weak disorder regime $\beta<\beta_{\mathrm{c}}$ and note that

$$
Z_{n}(\beta)=2^{n} \phi(\beta)^{n} M_{n}^{(\beta)}(\rho) \sim 2^{n} \phi(\beta)^{n} M^{(\beta)}(\rho),
$$

and from this we readily get the weak disorder part of Theorem 2. For every vertex $v \in T$ we denote by $B(v) \subset \partial T$ the set of all rays passing through the vertex $v$. The collection $(B(v): v \in T)$ is exactly the collection of all balls in $\partial T$. We obtain, with $m:=n-|v| \geq 0$,

$$
\begin{aligned}
\mu_{n}^{(\beta)}(B(v)) & =\frac{1}{Z_{n}(\beta)} \sum_{\substack{w \in T(v) \\
|w|=n}} \exp \left\{\beta \sum_{j=1}^{n} V\left(w_{j}\right)\right\} \\
& =\frac{1}{Z_{n}(\beta)} 2^{m} \phi(\beta)^{m} \exp \left\{\beta \sum_{j=1}^{|v|} V\left(v_{j}\right)\right\} M_{m}^{(\beta)}(v),
\end{aligned}
$$

and combining the last two displays we get

$$
\lim _{n \rightarrow \infty} \mu_{n}^{(\beta)}(B(v))=2^{-|v|} \phi(\beta)^{-|v|} \exp \left\{\beta \sum_{j=1}^{|v|} V\left(v_{j}\right)\right\} \frac{M^{(\beta)}(v)}{M^{(\beta)}(\rho)} .
$$

This suffices to ensure, for every $0<\beta<\beta_{\mathrm{c}}$, the almost sure existence of the infinite volume Gibbs measure $\mu^{(\beta)}$, which is characterised by

$$
\mu^{(\beta)}(B(v))=2^{-|v|} \phi(\beta)^{-|v|} \exp \left\{\beta \sum_{j=1}^{|v|} V\left(v_{j}\right)\right\} \frac{M^{(\beta)}(v)}{M^{(\beta)}(\rho)} .
$$

The key to Theorem 3 is now that, other than in some otherwise similar models such as the random energy model discussed in [6], the measures $\mu_{\beta}$ are fractal measures in the sense that they are supported by a very thin subset of $\partial T$.

This can be explored using the method of spined trees in a way similar to the previous example: Let $\mathrm{P}$ the distribution of the environment $\mathcal{V}$ and, given $\mathcal{V}$, we select the spine according to the infinite volume Gibbs measure $\mu_{\mathcal{V}}^{(\beta)}$ (indicating the dependence on the environment by an additional subindex). Slightly abusing the notation of the previous example, we let SpinedTrees be 
the space of weights attached to the vertices of a binary tree with a marked spine. Recall the definition of the canonical shift $\theta$ on SpinedTrees.

Writing $M_{\mathcal{V}}^{(\beta)}$ instead of $M^{(\beta)}(\rho)$, the measure on SpinedTrees given by

$$
\mu_{\mathcal{V}}^{(\beta)}(d v) M_{\mathcal{V}}^{(\beta)} \mathrm{P}(d \mathcal{V})
$$

is shift-invariant and ergodic. From the ergodic theorem we thus obtain

$$
\lim _{n \rightarrow \infty} \frac{1}{n} \sum_{j=1}^{n} V\left(v_{j}\right)=\int V(\rho) M_{\mathcal{V}}^{(\beta)} \mathrm{P}(d \mathcal{V})=\frac{\phi^{\prime}(\beta)}{\phi(\beta)}=a(\beta),
$$

and, recalling the representation of the infinite volume Gibbs measure,

$$
\begin{aligned}
\lim _{n \rightarrow \infty} & \frac{-1}{n} \log \mu^{(\beta)}\left(B\left(v_{n}\right)\right) \\
\quad & \log 2+\log \phi(\beta)-\int\left(\beta V(\rho)+\log \frac{M^{(\beta)}\left(v_{1}\right)}{M^{(\beta)}(\rho)}\right) \mu_{\mathcal{V}}^{(\beta)}(d v) M_{\mathcal{V}}^{(\beta)} \mathrm{P}(d \mathcal{V}) \\
& =\log 2+\log \phi(\beta)-\beta a(\beta),
\end{aligned}
$$

for $\mu^{(\beta)}$-almost every $v \in \partial T$ and P-almost every medium $\mathcal{V}$.

For the proof of Theorem 3 (a) we use Egorov's theorem to select a compact set $A \subset \partial T$ such that $\mu^{(\beta)}(A)>0$ and the two convergences just proved hold uniformly for all rays in $A$. We then define the tree

$$
\tilde{T}=\bigcup_{v \in A} \bigcup_{j=1}^{\infty}\left\{v_{j}\right\} \subset T .
$$

Note that, by compactness of $A$, we have $\partial \tilde{T}=A$. The second convergence readily ensures that

$$
\operatorname{dim}(\tilde{T})=\log 2+\log \phi(\beta)-\beta a(\beta)=f(\beta),
$$

and, using the first convergence, we get

$$
\lim _{n \rightarrow \infty} \frac{1}{\beta n} \log \sum_{\substack{v \in \tilde{T} \\|v|=n}} \exp \left\{\beta \sum_{j=1}^{n} V\left(v_{j}\right)\right\}=\frac{f(\beta)}{\beta}+a(\beta)=h(\beta)
$$

and this completes the sketch of the proof of Theorem 3 (a).

The consideration of the infinite volume Gibbs measures $\mu^{(\beta)}$ also establishes the lower bound in the 'crude spectrum'

$$
\lim _{n \rightarrow \infty} \frac{1}{n} \log \#\left\{v \in T:|v|=n, \sum_{j=1}^{n} V\left(v_{j}\right) \geq n a(\beta)\right\}=f(\beta),
$$

while the corresponding upper bound follows easily from Cramér's theorem. Theorem 3 (b) can now be established by studying for any $\eta<f(\beta)$ the 'worst case scenario' of a tree $\tilde{T} \subset T$ with $\operatorname{dim}(\tilde{T})<\eta$, which captures in each generation $n$ the maximal possible value of $\sum \exp \left\{\beta \sum V\left(v_{j}\right)\right\}$. 


\section{The speed of emergence in Kingman's coalescent}

Kingman's coalescent is probably the most studied object in mathematical genetics and the key problems in this area are certainly more fundamental than the study of multifractal spectra. However, there are also some mathematical aspects on which a multifractal spectrum can shed some light.

We start the investigation from a simple population model. Suppose first that the population consists of $n$ individuals positioned at $\{1, \ldots, n\}$. Each position $i$ carries an independent exponential clock with rate $(n-1) / 2$ and, once this clock rings, the individual at position $i$ produces two offspring, one at position $i$, the other one at a position $j \in\{1, \ldots, n\} \backslash\{i\}$ chosen uniformly at random. At the same time the individual that used to be at position $j$ dies.

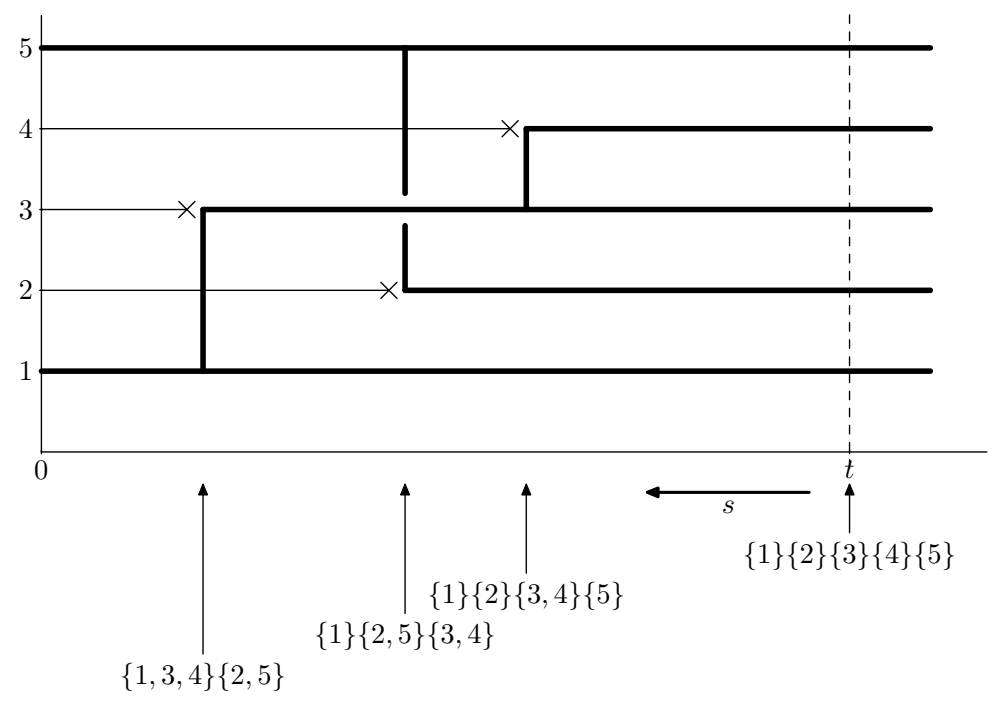

Figure 5: An illustration of Kingman's coalescent restricted to 5 individuals. The population evolves from left to right, a cross at position $j$ indicates that the particle in the position $j$ dies and is replaced by the offspring of the particle in the position connected to the cross by a vertical line. The coalescent is obtained by looking from right to left.

For any fixed time $t>0$ this population model gives rise to a natural Markov process of ancestral partitions $\left(\Pi_{s}^{(n)}: 0 \leq s \leq t\right)$ called the $n$-coalescent. It takes values in the space of partitions of $\{1, \ldots, n\}$ and we declare $i, j \in\{1, \ldots, n\}$ to be in the same partition set of $\Pi_{s}^{(n)}$ if the individuals in position $i$ and $j$ at time $t$ have the same ancestor at time $t-s$. Note that this process starts at time $s=0$ with the trivial partition consisting entirely of singletons.

Kingman [14] has shown that there exists a unique Markov process $\left(\Pi_{s}: s \geq 0\right)$ with values in the space of partitions of $\mathbb{N}$ such that, for all $n \in \mathbb{N}$ and $t>0$, the process running for $t$ time units obtained by restricting partitions to $\{1, \ldots, n\}$ is an $n$-coalescent. This process is called Kingman's coalescent. 
One of the key features of Kingman's coalescent is that it comes down from infinity, which means that for every time $s>0$ the number of partition sets, or blocks, in $\Pi_{s}$ is almost surely finite. Define the frequency of a block $B \subset \mathbb{N}$ as

$$
\lim _{n \rightarrow \infty} \frac{1}{n} \#(B \cap\{1, \ldots, n\}) .
$$

At all times $s>0$ all blocks of $\Pi_{s}$ have positive frequency, almost surely. In order to understand the instant transition from a state of dust at time $s=0$, when all blocks are singletons, to a state at time $s>0$ when the partition consists of finitely many blocks of positive frequency, we would like to follow the sequences of nested blocks as $s \downarrow 0$, and study the possible rates of decrease of the block frequencies in form of a multifractal spectrum.

We need to rigorously define a metric space representing the sequences of nested blocks. This construction is due to Evans [9]. We first define a (random) metric on $\mathbb{N}$ by letting

$$
\delta(i, j)=\inf \left\{s>0: i, j \in B \text { for some } B \in \Pi_{s}\right\} .
$$

The required metric space $(\mathcal{S}, \delta)$ is the completion of $(\mathbb{N}, \delta)$. Indeed, the set $\mathcal{S}$ is simply the boundary of a rooted, binary tree and all the interesting random structure enters in the metric $\delta$. It is shown in [9] that $\operatorname{dim} \mathcal{S}=1$.

Given an element $x \in \mathcal{S}$ we need to make sense of the speed of coalescence at $x$. For this purpose we define a probability measure $\eta$ on $(\mathcal{S}, \mathcal{B}$ orel $)$ by letting

$$
\eta(B(x, s))=\lim _{n \rightarrow \infty} \frac{1}{n} \#\{i \in\{1, \ldots, n\}: \delta(i, x) \leq s\} .
$$

This uniquely defines a probability measure $\eta$ on $\mathcal{S}$. We define the lower and upper speed of emergence of $x \in \mathcal{S}$ as

$$
\underline{\operatorname{speed}}(x)=\liminf _{s \downarrow 0} \frac{\log \eta(B(x, s))}{\log s}, \quad \overline{\operatorname{speed}}(x)=\limsup _{s \downarrow 0} \frac{\log \eta(B(x, s))}{\log s} .
$$

From the results of [9] we infer that, almost surely,

$$
\underline{\operatorname{speed}}(x)=\overline{\operatorname{speed}}(x)=1,
$$

for $\eta$-almost every $x$. The multifractal spectrum shows the presence of points of exceptional upper speed of emergence.

Theorem 4 (Berestycki et al.). Almost surely, if $1 \leq a \leq 2$, then

$$
\operatorname{dim}\{x \in \mathcal{S}: \overline{\operatorname{speed}}(x) \geq a\}=\frac{2}{a}-1,
$$

and there are no points $x \in \mathcal{S}$ with $\overline{\operatorname{speed}}(x)>2$ or $\overline{\operatorname{speed}}(x)<1$. 


\section{Remark:}

(a) This is a very different kind of spectrum compared to those in the previous sections, as variations in the speed only happen at exceptional times $s>0$ and lower speeds exceeding one can not occur. Spectra of this kind have been associated with the 'breakdown of the multifractal formalism' in various examples, see e.g. [20] or [15].

(b) It is conjectured that the lower speed of emergence equals one for all $x \in \mathcal{S}$, which would be in contrast with the case of Beta-coalescents with parameter $1<\alpha<2$. In that case exceptionally small lower speeds can occur, see [2, Theorem 5.1].

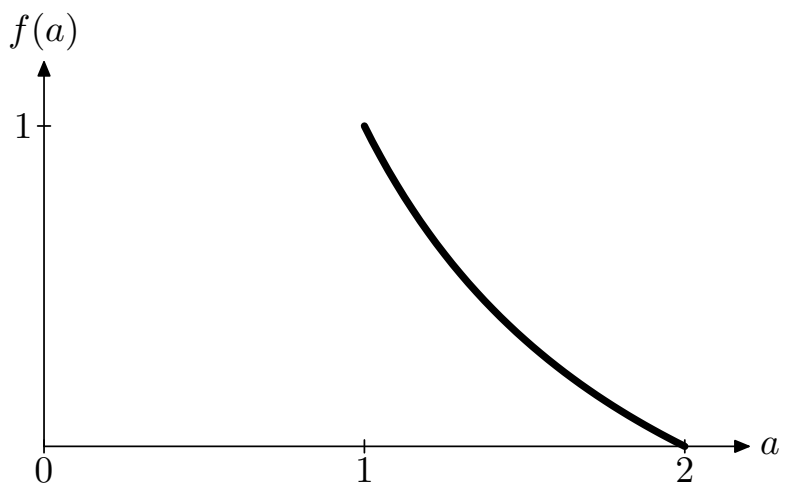

Figure 6: The dimension spectrum for the upper speed of emergence in Kingman's coalescent.

\section{The idea of the proof of Theorem 4}

The key to the proof is to establish a link between the metric space $\mathcal{S}$ equipped with the measure $\eta$ and a tree of excursions embedded in a Brownian motion equipped with a local time measure. The multifractal structure of the local time can then be analysed, for example using a percolation technique.

For an (unfortunately very superficial) sketch of this link, we start with a Brownian motion $\{B(t): 0 \leq t \leq \tau\}$ stopped at

$$
\tau=\inf \left\{t>0: L^{0}(t)=1\right\},
$$

where $\left\{L^{s}(t): t \geq 0\right\}$ is the local time process at level $s$. Write $Z_{t}=L^{t}(\tau)$ and recall from the Ray-Knight theorem that $\left\{Z_{t}: t \geq 0\right\}$ is a Feller diffusion. Then define an increasing process $\{R(t): 0 \leq t<T\}$ by

$$
R(t)=\int_{0}^{t} \frac{1}{Z_{s}} d s
$$


Note that this process explodes at time $T=\max _{0 \leq t \leq \tau} B(t)$. Therefore its inverse $\left\{R^{-1}(t): t \geq 0\right\}$ maps the positive halfline onto $[0, T)$. For any $0<s<1$ we let $E(s)$ be the set of excursions of the Brownian motion above level $R^{-1}(s)$ that reach level $R^{-1}(1)$. Note that $E(s)$ is a finite set, but the number of elements in $E(s)$ is increasing to infinity as $s \uparrow 1$.

We denote by $\Xi$ the set of functions $(\zeta(s): 0 \leq s<1)$ such that

- $\zeta(s) \in E(s)$ for any $0 \leq s<1$,

- $\zeta\left(s_{2}\right)$ is contained in $\zeta\left(s_{1}\right)$ for any $0<s_{1}<s_{2}<1$.

$\Xi$ is a complete metric space when endowed with the metric

$$
d\left(\zeta_{1}, \zeta_{2}\right)=\inf \left\{1-s: \zeta_{1}(s)=\zeta_{2}(s)\right\}
$$

and there exists a metric isomorphism $\Phi: \Xi \rightarrow \mathcal{S}$, such that

$$
\eta(B(\Phi(\zeta), s))=Z_{R^{-1}(1)}^{-1} \ell(\zeta(1-s)) \quad \text { for all } \zeta \in \Xi \text { and } 0<s<1,
$$

where $\ell(e)$ denotes the local time of the excursion $e \in E(1-s)$ at level $R^{-1}(1)$.

We now rescale the paths $(\zeta(s): 0<s<1)$ by letting

$$
\zeta^{\prime}(s)=\zeta\left(R\left(s R^{-1}(1)\right)\right) \quad \text { for } 0<s<1,
$$

so that $\zeta^{\prime}(s)$ is an excursion above level $s R^{-1}(1)$ which reaches level $R^{-1}(1)$. Note that the mapping $\psi: \Xi \rightarrow \Xi^{\prime}$ which maps every path $\zeta$ to its rescaling $\zeta^{\prime}$ is a bijection, but does not preserve the metric $d$. However, because

$$
R^{-1}(1)-R^{-1}(1-t) \sim q t \quad \text { as } t \downarrow 0,
$$

for some (random) constant $q$, small distances are only linearly affected by $\psi$ and hence $\operatorname{dim}(\psi(A))=\operatorname{dim} A$ for any $A \subset \Xi$ and

$$
\overline{\text { speed }}(\Phi(\zeta))=\limsup _{s \downarrow 0} \frac{\log \ell\left(\zeta^{\prime}(1-s)\right)}{\log s} .
$$

Starting from this representation, the idea of the proof is to test for which deletion parameters the set of paths with high upper speed of emergence has a positive probability of surviving a (suitably defined) percolation process. This technique is the continuous time analogue of the percolation technique based on [16] and used for a similar problem in a discrete setup in [19].

To be precise, fix the deletion parameter $0<\lambda<1$. With any path $\left(\zeta^{\prime}: 0 \leq\right.$ $s \leq 1)$ we associate a Poison process, or clock, with intensity measure

$$
\lambda \frac{d s}{1-s} \quad \text { on }(0,1),
$$

which kills the path at the first strike of the clock. Any paths $\zeta_{1}^{\prime}, \zeta_{2}^{\prime} \in \Xi^{\prime}$, 
- share the same clock for $0<s \leq d\left(\zeta_{1}^{\prime}, \zeta_{2}^{\prime}\right)$, but

- have independent clocks for $d\left(\zeta_{1}^{\prime}, \zeta_{2}^{\prime}\right)<s<1$.

We say that a set $A \subset \Xi^{\prime}$ of paths survives percolation, if there exists a path in $A$, which has lifetime one. Then any analytic set has positive probability of surviving the percolation if its Hausdorff dimension is $>\lambda$, but zero probability if it is $<\lambda$.

To find, for $a \geq 1$, the critical deletion parameter of the set

$$
\left\{\zeta^{\prime} \in \Xi^{\prime}: \limsup _{s \downarrow 0} \frac{\log \ell\left(\zeta^{\prime}(1-s)\right)}{\log s} \geq a\right\}
$$

one proves the following key estimate: For an excursion $e \in E(0)$,

$$
\lim _{x \downarrow 0} \frac{1}{\log x} \log \mathbb{P}\left(\{\ell(e) \leq x\} \cap\left\{\left\{\zeta^{\prime}: \zeta^{\prime}(0)=e\right\} \text { survives }\right\}\right)=1+\lambda .
$$

Roughly speaking, this holds because the typical local time of an excursion above level $1-x$ which reaches height one is of order $x$. Therefore the optimal strategy to obtain the event $\{\ell(e)<x\}$ is to ensure that only one of the excursions above level $1-x$, which are embedded in $e$, reaches height one. The probability of this event is of order $x$ and, given this, the probability that the set of paths starting with $\zeta^{\prime}(0)=e$ survives percolation is, up to a constant multiple, equal to the probability that the path $\left(\zeta^{\prime}(s): 0<s<1-x\right)$ survives, which is

$$
\exp \left\{-\lambda \int_{0}^{1-x} \frac{d s}{1-s}\right\}=x^{\lambda}
$$

With the key estimate at hand we roughly argue as follows: Typically the number of excursions above level $1-s$ that reach level 1 is of order $1 / s$. Out of the paths $\left(\zeta^{\prime}(t): 0 \leq t \leq 1-s\right)$ ending in these excursions typically a proportion of order $s^{\lambda}$ survives up to this time. Hence, for a fixed small $s>0$, we can expect of order $s^{\lambda-1}$ conditionally independent trials to realise the event

$$
\left\{\ell\left(\zeta^{\prime}(1-s)\right) \leq s^{a}\right\} \cap\left\{B\left(\zeta^{\prime}, s\right) \text { survives }\right\} .
$$

By scaling the key estimate, this event has probability of order

$$
\left(\frac{s^{a}}{s}\right)^{1+\lambda}=s^{(a-1)(1+\lambda)} .
$$

Hence, the expected number of paths $\left(\zeta^{\prime}(t): 0 \leq t \leq 1-s\right)$, which survive the percolation procedure and satisfy $\ell\left(\zeta^{\prime}(1-s)\right) \leq s^{a}$ is

$$
s^{\lambda-1} s^{(a-1)(1+\lambda)},
$$

indicating that the threshold for the existence of paths of length one occurs when $\lambda-1+(a-1)(1+\lambda)=0$ or, equivalently, when $\lambda=2 / a-1$. This argument readily gives the upper bound in Theorem 4 , while the lower bound follows with only marginally more effort, exploiting the self-similarity of the Brownian structure by means of Baire's theorem. 


\section{Conclusion}

We have reviewed different forms of multifractal spectra using some simple but interesting examples. At least for me, the spectra (and their derivation) have been helpful in forming an intuition for the studied processes. Many of the martingale and tree ideas used in our proofs can be considered as part of the folklore and have been rediscovered many times in different guises. While it is impossible to give a full list of the relevant publications here, $[4,10,11,12,13,16,18,21]$ represents a good selection of the pioneering papers, from which these ideas have been formed.

\section{References}

[1] BerestyCKI, J. Multifractal spectra of fragmentation processes. J. Stat. Phys. 113 100-111 (2002).

[2] Berestycki, J.; Berestycki, N.; Schweinsberg, J. Beta-coalescents and continuous stable trees. Ann. Probab. 35 1835-1887 (2007).

[3] Bertoin, J. Random fragmentation and coagulation processes. Cambridge University Press (2006).

[4] Biggins, J. D. Martingale convergence in the branching random walk. J. Appl. Probab. 14 25-37 (1977).

[5] Birkner, M.; Blath, J.; Capaldo, M.; Etheridge, A.; Möhle, M.; Schweinsberg, J.; Wakolbinger, A. Alpha-stable branching and beta-coalescents. Electron. J. Probab. 10 303-325 (2005).

[6] Bovier, A. Statistical mechanics of disordered systems. Cambridge University Press (2006).

[7] Buffet, E.; Patrick, A.; Pulé, J.V. Directed polymers on trees: a martingale approach. J. Phys. A 26 1823-1834 (1993).

[8] Derrida, B.; Spohn, H. Polymers on disordered trees, spin glasses, and traveling waves. J. Stat. Phys. 51 817-840 (1988).

[9] Evans, S. N. Kingman's coalescent as a random metric space. In: Stochastic models (Ottawa, 1998), CMS Conf. Proc., 26, Amer. Math. Soc., Providence, 105-114 (2000).

[10] Hawkes, J. Trees generated by a simple branching process. J. London Math. Soc. 24 373-384 (1981). 
[11] Joffe, A.; Le Cam, L.; Neveu, J. Sur la loi des grands nombres pour des variables aléatoires de Bernoulli attachées à un arbre dyadique. C. R. Acad. Sci. Paris 277 A963-A964 (1973).

[12] Kahane, J.-P.; Peyrière, J. Sur certaines martingales de Benoit Mandelbrot. Adv. Math. 22 131-145 (1976).

[13] Kingman, J. F. C. The first birth problem for an age-dependent branching process. Ann. Probab. 3 790-801 (1975).

[14] Kingman, J.F.C. The coalescent. Stoch. Proc. Appl. 13 235-248 (1982).

[15] Klenke, A.; Mörters, P. The multifractal spectrum of Brownian intersection local time. Ann. Probab. 33 1255-1301 (2005).

[16] Lyons, R. Random walks and percolation on trees. Ann. Probab. 18 931958 (1990).

[17] LyOns, R. A simple path to Biggins' martingale convergence for branching random walks. In: Classical and modern branching processes, K. Athreya and P. Jagers (Eds), Springer, New York, 217-222 (1997).

[18] Lyons, R.; Pemantle, R.; Peres, Y. Ergodic theory on Galton-Watson trees: speed of random walk and dimension of harmonic measure. Ergodic Theory Dynam. Systems 15 593-619 (1995).

[19] Mörters, P.; Shieh, N.R. On the multifractal spectrum of the branching measure on a Galton-Watson tree. J. Appl. Probab. 41 1223-1229 (2004).

[20] Perkins, E.; TAYlor, S.J. The multifractal structure of super-Brownian motion. Ann. Inst. Henri Poincaré 34 97-138 (1998).

[21] Waymire, E.; Williams, S.C. A general decomposition theory for random cascades. Bull. Amer. Math. Soc. 31 216-222 (1994). 